\title{
On equations with rotations
}

\section{PRZEWORSKA-R OLEWICZ (Warszawa)}

If an equation contains together with the unknown function $x(t)$ of a complex variable the values $x\left(\varepsilon_{1} t-\alpha_{1}\right), \ldots, x\left(\varepsilon_{N} t-\alpha_{N}\right)$, where $\varepsilon_{1}, \ldots, \varepsilon_{N}$ are $N$-th roots of the unity, $\alpha_{1}, \ldots, \alpha_{N}$ are complex numbers, then it will be called equation with rotation.

The case $N=2, a_{1}=\ldots=a_{N}=0$, was solved completely in paper [3] on equations with reflection. The purpose of this paper is to solve equations with rotation (for some $\alpha_{k}$ ). The method is based on properties of involutions of order $N$ (see [1]).

An ordinary differential equation with rotation will be considered as an example.

1. Let $S$ be an involution of order $N$, i.e. a linear operator acting in a linear space $X$ (over complex scalars) such that

$$
S^{N}=I \text {, }
$$

where $I$ denotes the identity operator, $N \geqslant 2$, and there is no polynomial $P(t)$ of order less than $N$ such that $P(S)=0$. The following properties of involution of order $N$, proved in [1] (see also [3]), will be used. Let us write

$$
P_{v}=\frac{1}{N} \sum_{k=0}^{N-1} \varepsilon^{-k v} S^{\dot{k}}, \quad v=1,2, \ldots, N
$$

where $\varepsilon=e^{2 \pi i / N}$.

Since $\varepsilon$ is the $N$-th root of the unity (with the smallest argument), we have $\varepsilon \neq 1$ and

(1.3) $\quad \varepsilon^{N}=1, \quad \varepsilon^{-k}=\varepsilon^{N-k}, \quad \sum_{k=0}^{N-1} \varepsilon^{k m}=\left\{\begin{array}{l}0 \text { for } m=1,2, \ldots, N-1 \\ N \text { for } m=N\end{array}\right.$

The operators $P_{v}$ are disjoint projectors giving a partition of unity:

$$
P_{\nu} P_{\mu}=\delta_{p \mu} P_{\nu}, \quad \sum_{\nu=1}^{N} P_{\nu}=I
$$

where $\delta_{\nu \mu}$ is the Kronecker symbol. Moreover,

$$
S P_{v}=\varepsilon^{\nu} P_{v} \quad(v=1,2, \ldots, N) .
$$


From this it follows that the space $X$ can be decomposed in a direct

$$
X=X_{(1)} \oplus \ldots \oplus X_{(N)}
$$

of spaces $X_{(v)}$ such that

(1.7). $\quad X_{(v)}=P_{p} X$ and $S x=\varepsilon^{v} x$ for $x \in X_{(v)} \quad(v=1,2, \ldots, N)$ and every element $x \in X$ can be written in a unique manner in the form

$$
x=x_{(1)}+\ldots+x_{(N)}, \quad \text { where } x_{(v)} \in X_{(v)},
$$

if we put $x_{(v)}=P_{\nu} x(\nu=1,2, \ldots, N)$.

A linear operator transforming $X$ into itself is permuting an involution $S$ of order $N$ acting in $X$ if both superpositions $S D$ and $D S$ exist and

$$
D S=\varepsilon S D, \quad \text { where } \varepsilon=e^{2 \pi i / N} .
$$

It will be shown further that a permuting operator $D$ and its powers permute the spaces $X_{(1)}, \ldots, X_{(N)}$ determined by decomposition (1.6).

PROPERTY 1.1. For arbitrary positive integers $k$ and $m$, if $D$ is permuting an involution $S$ of order $N$, then

$$
D^{m} S^{k}=\varepsilon^{m k} S^{k} D^{m}
$$

Pro of (by induction). By assumption, (1.10) is true for $m=k=1$. Let us suppose $(1.10)$ be true for $m=1$. Then $D S^{k+1}=(D S) S^{k+1}$ $=\varepsilon S\left(D S^{k}\right)=\varepsilon \varepsilon^{k} D S^{k+1}=\varepsilon^{k+1} S^{k+1} D$. Let $k$ be arbitrarily fixed. Then, supposing (1.10) to be true, we obtain

$$
\begin{aligned}
D^{m+1} S^{k} & =D\left(D^{m} S^{k}\right)=D\left(\varepsilon^{k m} S^{k} D^{m}\right)=\varepsilon^{k m}\left(D S^{k}\right) D^{m} \\
& =\varepsilon^{k m} \varepsilon^{k} S^{k} D^{m+1}=\varepsilon^{k(m+1)} S^{k} D^{m+1} .
\end{aligned}
$$

PROPERTY 1.2. If $D$ is permuting an involution $S$ of order $N$, then

$$
\begin{aligned}
P_{\nu} D & =D P_{v+1} \quad \text { for } v=1,2, \ldots, N-1, \\
P_{N} D & =D P_{1} .
\end{aligned}
$$

Proof. By definition, we have for $v=1,2, \ldots, N$

$$
\begin{aligned}
P_{v} D & =\left(\frac{1}{N} \sum_{k=0}^{N-1} \varepsilon^{-k v} S^{k k}\right) D=\frac{1}{N} \sum_{k=0}^{N-1} \varepsilon^{-k v} S^{k} D=\frac{1}{N} \sum_{k=0}^{N-1} \varepsilon^{-k v} \varepsilon^{-k} D S^{k} \\
& =D \frac{1}{N} \sum_{k=0}^{N-1} \varepsilon^{-k(\nu+1)} S^{k}
\end{aligned}
$$

and for $v=N$ we find $\varepsilon^{-k(N+1)}=\varepsilon^{-k}$. Hence $P_{N} D=D P_{1}$. For $v=1,2$, $\ldots, N-1$ we obtain $P_{v} D=D P_{v+1}$.
Similarly we prove the following

PROPERTY 1.3. Under assumptions of 1.2 ,

$$
\begin{gathered}
P_{y} D^{2}=D^{2} P_{v+2} \quad \text { for } v=1,2, \ldots, N-2, \\
P_{N-1} D^{2}=D^{2} P_{1}, \quad P_{N} D^{2}=D^{2} P_{2} .
\end{gathered}
$$

PROPERTY 1.4. Under assumption of 1.2 , the operator $D^{N}$ is commuting with $S$ and $P_{\nu}$ :

$$
D^{N} S=S D^{N} \text { and } D^{N} P_{p}=P_{p} D^{N} \quad(v=1,2, \ldots, N) .
$$

Property 1.5. Let

$$
Q(t)=\sum_{k=0}^{N-1} q_{k} t^{k}
$$

be an arbitrary polynomial (of complex variable $t$ ) with constant complex coefficients. Let $S$ be an involution of order $N$. Then

$$
Q(S)=\sum_{y=1}^{N} Q\left(\varepsilon^{\nu}\right) P_{\nu} .
$$

Indeed, formulae (1.4) and (1.5) imply

$$
\begin{aligned}
Q(S) & =\sum_{k=0}^{N-1} q_{k} S^{k}=\sum_{k=0}^{N-1} q_{k} S^{k}\left(\sum_{\nu=1}^{N} P_{\nu}\right) \\
& =\sum_{\nu=1}^{N}\left(\sum_{k=0}^{N-1} q_{k} S^{k} P_{\nu}\right)=\sum_{\nu=1}^{N} \sum_{k=0}^{N-1} q_{k} \varepsilon^{v / k} P_{\nu} \\
& =\sum_{\nu=1}^{N}\left[\sum_{k=0}^{N-1} q_{k}\left(\varepsilon^{\nu}\right)^{k}\right] P_{\nu}=\sum_{\nu=1}^{N} Q\left(\varepsilon^{\nu}\right) P_{\nu} .
\end{aligned}
$$

Property 1.6. Let

$$
Q(t)=\sum_{k=0}^{N-1} q_{k} t^{k}, \quad R(t)=\sum_{k=0}^{N-1} r_{k} t^{k}
$$

be arbitrary polynomials (of a complex variable $t$ ) with constant complex coefficients. Let $S$ be an involution of order $N$. Then

$$
Q(S) R(S)=\sum_{\nu=1}^{N} Q\left(\varepsilon^{\nu}\right) R\left(\varepsilon^{\nu}\right) P_{\nu} .
$$

Indeed, formula (1.4) and the preceding property imply

$$
\begin{aligned}
Q(S) R(S) & =\left[\sum_{\nu=1}^{N} Q\left(\varepsilon^{\nu}\right) P_{\nu}\right]\left[\sum_{\mu=1}^{N} R\left(\varepsilon^{\mu}\right) P_{\mu}\right] \\
& =\sum_{\nu, \mu=1}^{N} Q\left(\varepsilon^{\nu}\right) R\left(\varepsilon^{\mu}\right) P_{\nu} P_{\mu} \\
& =\sum_{\nu, \mu=1}^{N} Q\left(\varepsilon^{\nu}\right) R\left(\varepsilon^{\mu}\right) \delta_{\nu \mu} P_{\nu}=\sum_{\nu=1}^{N} Q\left(\varepsilon^{\nu}\right) R\left(\varepsilon^{\nu}\right) P_{\nu} .
\end{aligned}
$$


COROLLARY 1.1. Under the assumption of Property 1.6, $Q(S) R(S)=a I$, where $a$ is an arbitrary scalar, if and only if $Q\left(\varepsilon^{v}\right) R\left(\varepsilon^{v}\right)=a$ for $\nu=1$, $2, \ldots, N$.

Corollary 1.2. Under the assumption of Property 1.5 there exists $Q^{-1}\left(S^{\prime}\right)$ if and only if $Q\left(\varepsilon^{\nu}\right) \neq 0$ for $v=1,2, \ldots, N$ and

$$
Q^{-1}(S)=\sum_{\nu=1}^{N} Q^{-1}\left(\varepsilon^{\nu}\right) P_{\nu}
$$

This and Property 1.5 imply

$$
S^{k}=\sum_{\nu=1}^{N} \varepsilon^{p / k} P_{\nu} \quad \text { for } k=0, \pm 1, \pm 2, \ldots
$$

PROPERTy 1.7. Under the assumption of Property 1.5,

$$
Q\left(\varepsilon^{m} S^{S}\right)=\sum_{\nu=1}^{N} Q\left(\varepsilon^{v+m}\right) P_{\nu} \quad(m= \pm 1, \pm 2, \ldots) .
$$

Indeed,

$$
\begin{aligned}
Q\left(\varepsilon^{m} S\right) & =\sum_{k=0}^{N-1} q_{k}\left(\varepsilon^{m} S\right)^{k}=\sum_{k=0}^{N-1} q_{k} \varepsilon^{m k} S^{k}\left(\sum_{\nu=1}^{N} P_{\nu}\right) \\
& =\sum_{\nu=1}^{N}\left(\sum_{k=0}^{N-1} q_{k} \varepsilon^{m k} S^{k} P_{\nu}\right)=\sum_{\nu=1}^{N}\left(\sum_{k=0}^{N-1} q_{k} \varepsilon^{m k} \varepsilon^{v k} P_{\nu}\right) \\
& =\sum_{\nu=1}^{N}\left(\sum_{k=0}^{N-1} q_{k} \varepsilon^{(v+m) k}\right) P_{\nu}=\sum_{\nu=1}^{N} Q\left(\varepsilon^{\nu+m}\right) P_{\nu} .
\end{aligned}
$$

Property 1.8. Onder the assumption of Property 1.5, if $D$ is permuting $S$, then

$$
D Q(S)=Q(\varepsilon S) D
$$

Indeed, according to Property 1.2,

$$
\begin{aligned}
D Q(S) & =D \sum_{\nu=1}^{N} Q\left(\varepsilon^{\nu}\right) P_{v}=\sum_{\nu=1}^{N} Q\left(\varepsilon^{\nu}\right) D P_{\nu}=Q\left(\varepsilon^{\nu}\right) D P_{1}+\sum_{\nu=2}^{N} Q\left(\varepsilon^{\nu}\right) D P_{\nu} \\
& =Q(\varepsilon) P_{N} D+\sum_{\nu=2}^{N} Q\left(\varepsilon^{\nu}\right) P_{\nu-1} D=\left[Q\left(\varepsilon^{N+1}\right) P_{N}+\sum_{\mu=1}^{N-1} Q\left(\varepsilon^{\mu+1}\right) P_{\nu}\right] D \\
& =\left[\sum_{\nu=1}^{N} Q\left(\varepsilon^{\nu+1}\right) P_{\nu}\right] D=Q(\varepsilon S) D .
\end{aligned}
$$

COROLLARY 1.3. Under the assumption of Property 1.5, if $D$ is permuting $S$, then

$$
\begin{aligned}
& D^{m} Q(S)=Q\left(\varepsilon^{m} S\right) D \quad \text { for } m=1,2, \ldots, N-1 \\
& D^{N} Q(S)=Q(S) D^{N} .
\end{aligned}
$$

2. For any linear operator $T$ transforming a linear space $X$ into itself we denote by $D_{T}$ the domain of $T$ and by $Z_{T}$ the kernel of $T: Z_{T}$ $=\left\{x \in D_{T}: T x=0\right\}$.

Let $S$ be an involution of order $N$ acting in a linear space $X$ and let $D$ be permuting $S$. Let us consider the operator

$$
A=a(S)-b(S) D
$$

where

$$
a(t)=\sum_{k=0}^{N-1} a_{k} t^{k} \quad \text { and } \quad b(t)=\sum_{k=0}^{N-1} b_{k} t^{k}
$$

are arbitrary polynomials with constant complex coefficients. In this section we assume that

$$
a\left(\varepsilon^{p}\right) \neq 0 \text { and } b\left(\varepsilon^{p}\right) \neq 0 \text { for } v=1,2, \ldots, N .
$$

Under these assumptions we shall determine the set $Z_{A}$.

LEMMA 2.1. The equation $A x=0$ is equivalent to the following system of equations:

$$
\begin{aligned}
& D x_{(1)}=c_{N} x_{(N)}, \\
& D x_{(m+1)}=c_{m} x_{(m)} \quad(m=1,2, \ldots, N-1),
\end{aligned}
$$

where $x_{(m)}=P_{m} x$ and $c_{m}=a\left(\varepsilon^{m}\right) / b\left(\varepsilon^{m}\right) \neq 0$ (by assumption) for $m=$ $=1,2, \ldots, N$.

Proof. Property 1.5 and (1.4) imply

$$
\begin{aligned}
P_{m} a(S) & =P_{m} \sum_{\nu=1}^{N} a\left(\varepsilon^{v}\right) P_{\nu}=\sum_{\nu=1}^{N} a\left(\varepsilon^{\nu}\right) P_{m} P_{\nu} \\
& =\sum_{\nu=1}^{N} a\left(\varepsilon^{\nu}\right) \delta_{m \nu} P_{m}=a\left(\varepsilon^{m}\right) P_{m} .
\end{aligned}
$$

Similarly, $P_{m} b(S)=b\left(\varepsilon^{m}\right) P_{m}$. Hence $P_{m} A=P_{m}[a(S)-b(S) D]=P_{m} a(S)-P_{m} b(S) D=a\left(\varepsilon^{m}\right) P_{m}-b\left(\varepsilon^{m}\right) P_{m} D$ and, by Property 1.2,

(2.3) $\quad P_{m} A= \begin{cases}a\left(\varepsilon^{m}\right) P_{m}-b\left(\varepsilon^{m}\right) P_{m+1} & \text { for } m=1,2, \ldots, N-1, \\ a\left(\varepsilon^{N}\right) P_{N}-b\left(\varepsilon^{N}\right) D P_{1} & \text { for } m=N .\end{cases}$

Applying formulae (1.6), (1.7) and (1.8), we infer that the equation $A x=0$ is equivalent to the system of equations

$$
P_{m} A x=0 \quad(m=1,2, \ldots, N) .
$$


According to $\left(2.3^{\prime}\right)$; the last system can be written as follows:

$$
\begin{gathered}
{\left[a\left(\varepsilon^{m}\right) P_{m}-b\left(\varepsilon^{m}\right) D P_{m_{+1}}\right] x=0 \quad \text { for } m=1,2, \ldots, N-1,} \\
{\left[a\left(\varepsilon^{N}\right) P_{N}-b\left(\varepsilon^{N}\right) D P_{1}\right] x=0 \quad \text { for } m=N .}
\end{gathered}
$$

Since $P_{m} x=x_{(m)}$ and $b\left(\varepsilon^{m}\right) \neq 0$ for $m=1,2, \ldots, N$, we obtain finally the system $(2.2)$.

LEMMA 2.2. $Z_{A} \subset Z_{D^{N}-\lambda I}$, where

$$
\lambda=c_{1} c_{2} \ldots c_{N}=\prod_{1 \leqslant \nu \leqslant N} \frac{a\left(\varepsilon^{\nu}\right)}{b\left(\varepsilon^{\nu}\right)} \neq 0 .
$$

Proof. Let $x \in Z_{A}$, i.e. $A x=0$. According to Lemma 2.1, the equation $A x=0$ is equivalent to the system (2.2). Let us consider $x_{(1)}=P_{1} x$. From the system (2.2) we obtain

$$
\begin{aligned}
& D x_{(1)}=c_{N} x_{(N)}, \\
& D^{2} x_{(1)}=D\left(D x_{(1)}\right)=c_{N} D x_{(N)}=c_{N} c_{N-1} x_{(N-1)} \\
& D^{3} x_{(1)}=D\left(D^{2} x_{(1)}\right)=c_{N} c_{N-1} D x_{(N-1)}=c_{N} c_{N-1} c_{n-2} x_{(N-2)} \text {, } \\
& \ldots \ldots \ldots \ldots \ldots \ldots \ldots \ldots \ldots \ldots \ldots \ldots \ldots \ldots \ldots \\
& D^{N} x_{(1)}=D\left(D^{N-1} x_{(1)}\right)=c_{N} c_{N-1} \ldots c_{2} D x_{(2)}=c_{N} c_{N-1} \ldots c_{2} c_{1} x_{(1)}=\lambda x_{(1)} .
\end{aligned}
$$

Hence $\left(D^{N}-\lambda I\right) x_{(1)}=0$ and $x_{(1)} \in Z_{D^{N}-\lambda I}$. Similarly, we can show that $x_{(m)}=P_{m} x \in Z_{D^{N}-\lambda I}(m=2,3, \ldots, N)$. Since $x_{(m)} \in X_{(m)}$ and the space $X$ is decomposed into a direct sum of spaces $X_{(m)}$, we obtain

$$
x=\sum_{m=1}^{N} x_{(m)} \epsilon Z_{D^{N}-\lambda I},
$$

which was to be proved.

LEMMA 2.3. $Z_{D^{N}-\lambda I}=\underset{0 \leqslant k \leqslant N-1}{\oplus} Z_{D-\lambda_{k} I}$, where $\lambda_{k}$ are $N$-th roots of $\lambda$ :

$$
\lambda_{k}=\sqrt[N]{|\lambda|} e^{(2 \pi k+\varphi) i / N},
$$

where $\varphi=\operatorname{Arg} \lambda(0 \leqslant \varphi \leqslant 2 \pi), k=0,1, \ldots, N-1$.

Proof. Let us remark that

$$
D^{N}-\lambda I=\left(D-\lambda_{0}\right)\left(D-\lambda_{1}\right) \ldots\left(D-\lambda_{N-1}\right) .
$$

The operator $D$ satisfies the polynomial identity $D^{N}-\lambda I=0$ on the space $Z_{D^{N}-\lambda I}$. Similarly as in (1.6), we can prove (see also [3], part A, Chapter II) that $Z_{D^{N}-\lambda I}=\underset{0 \leqslant k \leqslant N-1}{\oplus} Y_{l_{k}}$ and $y \in Y_{k}$ if and only if $D y=\lambda_{k} y$, because $\lambda_{0}, \ldots, \lambda_{N-1}$ are $N$-th roots of the equation $t^{N}-\lambda=0$. Therefore $Y_{k}=Z_{D-\lambda_{k} I}$ for $k=0,1, \ldots, N-1$.
THEOREM 2.1 .

$$
Z_{D^{N}-\lambda I}=\left\{z \in X: z=\sum_{k=0}^{N-1} \alpha_{k} S^{k} z_{k} ; z_{k} \in Z_{D-\lambda_{0} I}\right\}
$$

Pro of. First we remark that

$$
\lambda_{k}=\lambda_{0} \varepsilon^{k} \quad \text { for } k=1,2, \ldots, N-1 .
$$

Indeed,

$$
\lambda_{k}=\sqrt[N]{\lambda} e^{\frac{q+2 \pi k}{N} i}=\sqrt[N]{\lambda} e^{\frac{\varphi}{N} i}\left(e^{\frac{2 \pi i}{N}}\right)^{k}=\lambda_{0} \varepsilon^{k} \quad(k=1,2, \ldots, N-1) .
$$

Let us suppose that $z \in Z_{D-\lambda_{k} I}$. We show that $z=S^{k} u$, where $u \in Z_{D-\lambda_{0} I}$. Indeed,

$$
D z=\lambda_{k} z=\lambda_{0} \varepsilon^{k} z \quad \text { and } \quad S^{N-k} D z=\lambda_{0} \varepsilon^{k} S^{N-k} z .
$$

But Property 1.1 implies $S^{N-k} D z=\varepsilon^{-(N-k)} D S^{N-k} z$. Hence

$$
D S^{N-k} z=\lambda_{0} \varepsilon^{k} \varepsilon^{N-k} S^{N-k} z=\lambda_{0} S^{N-k} z .
$$

Therefore $u=S^{N-k} z \in Z_{D-\lambda_{0} I}$. But $z=S^{N} z=S^{k} S^{N-k} z=S^{k} u$. Indeed,

Conversely, we show that for any $z \in Z_{D-\lambda_{0} I}$ we have $S^{k} z \in Z_{D-\lambda_{K} I}$.

$$
D S^{k} z=\varepsilon^{k} S^{k} \dot{D} z_{0}=\varepsilon^{k} \lambda_{0} z=\lambda_{0} \varepsilon^{k} S^{k} z=\lambda_{k} S^{k} z .
$$

Hence $S^{k} z \in Z_{D-\lambda_{k}}$.

To find the general form of the set $Z_{A}$ we shall determine first this set in a particular case.

Proposition 2.2. If $\operatorname{dim} Z_{D-\lambda_{0} I}=1$, then

$$
\begin{aligned}
& Z_{A}=\left\{z \in X: z=d\left[\sum_{k=0}^{N-1} d_{k} S^{k}\right] z_{0} ; z_{0} \in Z_{D-\lambda_{0} I}\right. \text { and } \\
& \text { the scalar } \left.d \text { is arbitrary, } d_{k}=\sum_{m=1}^{N} \lambda_{0}^{-m} c_{1} c_{2} \ldots c_{m} V_{k, m}\right\},
\end{aligned}
$$

where by $V_{k, m}$ we denote the subdeterminant obtained by cancelling the $(k+1)$-th column and the $m$-th row of the Van der Monde determinant $V$ of numbers $\varepsilon^{2}, \varepsilon^{3}, \ldots, \varepsilon^{N}, \varepsilon^{1}$ and $c_{m}=a\left(\varepsilon^{m}\right) / b\left(\varepsilon^{m}\right)$.

Proof. Since $Z_{A} \subset Z_{D^{N}-\lambda I}\left(\right.$ Lemma 2.2 ) and $\operatorname{dim} Z_{D-\lambda_{0} I}=1$, we have $z \in Z_{A}$ if and only if

$$
z=\sum_{k=0}^{N-1} \alpha_{k} S^{\hbar} z_{0}, \quad z_{0} \in Z_{D-\lambda_{0} I},
$$


is arbitrary, and the coefficients $\alpha_{k}$ are chosen suitably. Let us write

$$
\alpha(S)=\sum_{k=0}^{N-1} a_{k} S^{k}
$$

Then $z=\alpha(S) z_{0}=\sum_{p=1}^{N} \alpha\left(\varepsilon^{v}\right) P_{p} z_{0}$ and $P_{m} z=\alpha\left(\varepsilon^{m}\right) P_{m} z_{0}$. Hence, by formula (2.3) in Lemma 2.1,

$$
\begin{gathered}
{\left[c_{m} \alpha\left(\varepsilon^{m}\right) P_{m}-\alpha\left(\varepsilon^{m+1}\right) D P_{m+1}\right] z_{0}=0, \quad m=1,2, \ldots, N-1,} \\
{\left[c_{N} \alpha\left(\varepsilon^{N}\right) P_{N}-\alpha(\varepsilon) D P_{1}\right] z_{0}=0,}
\end{gathered}
$$

where $c_{n}=a\left(\varepsilon^{m}\right) / b\left(\varepsilon^{n n}\right)$.

$$
\text { But }
$$

$$
\begin{gathered}
D P_{m+1} z_{0}=P_{m} D z_{0}=P_{m} \lambda_{0} z_{0}=\lambda_{0} P_{m} z_{0} \quad \text { for } m=1,2, \ldots, N-1, \\
D P_{1} z_{0}=P_{N} D z_{0}=P_{m} \lambda_{0} z_{0}=\lambda_{0} P_{N} z_{c} .
\end{gathered}
$$

Hence the last system can be written as follows:

$$
\begin{gathered}
{\left[c_{m} \alpha\left(\varepsilon^{m}\right)-\lambda_{0} \alpha\left(\varepsilon^{m+1}\right)\right] P_{m} z_{0} \quad(m=1,2, \ldots, N-1),} \\
{\left[a_{N} \alpha\left(\varepsilon^{N}\right)-\lambda_{0} \alpha(\varepsilon)\right] P_{N} z_{0}=0 .}
\end{gathered}
$$

Let us remarkitthat: $P_{m} z_{0} \neq 0$ for $m=1,2, \ldots, N$, if $z_{0} \neq 0$. Indeed, let us suppose that for an $m$ we have $P_{m} z_{0}=0$. This means that

$$
\sum_{k=0}^{N-1} \varepsilon^{-k m} S^{k} z_{0}=0
$$

but this implies linear dependence of all elements $z_{0}, S z_{0}, \ldots, S^{N-1} z_{0}$. But $S^{k} z_{0} \in Z_{D-\lambda_{k} I}$, and the space $Z_{D^{N}-\lambda I}$ is a direct sum of spaces $Z_{D \ldots . . k_{k} I}$; this implies $S^{k} z_{0}=0$ for $k=0,1, \ldots, N-1$. In particular, $z_{0}=0$, a contradiction. Hence, $z_{0}$ being arbitrary, Corollary 1.1 implies that equalities (2.7) hold if and only it

$$
\begin{gathered}
c_{m} \alpha\left(\varepsilon^{m}\right)-\lambda_{0} \alpha\left(\varepsilon^{m+1}\right)=0 \quad(m=1,2, \ldots, N-1), \\
c_{N} \alpha\left(\varepsilon^{N}\right)-\lambda_{n} \alpha(\varepsilon)=0 .
\end{gathered}
$$

We obtained finally the system of $N$ homogeneous equations with $N$ unknows $\alpha(\varepsilon), \alpha\left(\varepsilon^{2}\right), \ldots, \alpha\left(\varepsilon^{N}\right)$. The determinant $\Delta$ of this system is

$$
\Delta=\left|\begin{array}{rrrrrrr}
c_{1} & -\lambda_{0} & 0 & 0 & \ldots & 0 & 0 \\
0 & c_{2} & -\lambda_{0} & 0 & \ldots & 0 & 0 \\
0 & 0 & c_{3} & -\lambda_{0} & \ldots & 0 & 0 \\
\ldots & \ldots & \ldots & \ldots & \ldots & \ldots & \ldots \\
0 & 0 & 0 & 0 & \ldots & c_{N-1} & -\lambda_{0} \\
-\lambda_{0} & 0 & 0 & 0 & \ldots & 0 & c_{N}
\end{array}\right|
$$

The expansion of $\Delta$ with respect to the last row gives

$$
\begin{aligned}
& \Delta=(-1)^{N+1}\left(-\lambda_{0}\right)\left|\begin{array}{rrrrrr}
-\lambda_{0} & 0 & 0 & \ldots & 0 & 0 \\
c_{2} & -\lambda_{0} & 0 & \ldots & 0 & 0 \\
0 & c_{3} & -\lambda_{0} & \ldots & 0 & 0 \\
\ldots & \ldots & \ldots & \ldots & \ldots & \ldots \\
0 & 0 & 0 & \ldots & c_{N-1}-\lambda_{0}
\end{array}\right|+ \\
& +(1)^{2 N} c_{N}\left|\begin{array}{rrrrr}
c_{1} & -\lambda_{0} & 0 & \ldots & 0 \\
0 & c_{2} & -\lambda_{0} & \ldots & 0 \\
0 & 0 & c_{3} & \ldots & 0 \\
\ldots & \ldots & \ldots & \ldots & \ldots \\
0 & 0 & 0 & \ldots & c_{N}
\end{array}\right|
\end{aligned}
$$

Then first determinant has zeros only above the principal diagonal and the second one, only under the principal diagonal. Therefore.

because $\lambda_{0}^{N}=\lambda$.

$$
\begin{aligned}
\Delta & =(-1)^{N+1}\left(-\lambda_{0}\right)^{N}+(-1)^{2 N} c_{N} c_{1} c_{2} \ldots c_{N-1} \\
& =(-1)^{2 N+1} \lambda_{0}^{N}+\lambda=-\lambda+\lambda=0,
\end{aligned}
$$

Since the first subdeterminant of order $N-1$ is (by assumption) different from zero, we solve the system (2.8) by cancelling the last equation and by putting

$$
\alpha(\varepsilon)=\alpha,
$$

where $\alpha$ is an arbitrary complex number. We obtain

$$
\begin{aligned}
\alpha\left(\varepsilon^{2}\right) & =\frac{c_{1}}{\lambda_{0}} \alpha \\
\alpha\left(\varepsilon^{m+1}\right) & =\frac{c_{m}}{\lambda_{0}} \alpha\left(\varepsilon^{m}\right) \quad \text { for } m=2,3, \ldots, N-1 .
\end{aligned}
$$

Hence

$$
\alpha\left(\varepsilon^{m+1}\right)=\frac{c_{m} c_{m-1} \ldots c_{1}}{\lambda_{0}^{m}} \alpha,
$$

$\alpha$ is an arbitrary complex number $(m=1,2, \ldots, N-1)$.

We have determined

$$
\alpha\left(\varepsilon^{m+1}\right)=\sum_{k=0}^{N-1} \alpha_{k} \varepsilon^{(m+1)^{k}}
$$

Now we shall determine the constants $\alpha_{k}$. We obtain the following system of equations:

$$
\sum_{k=0}^{N-1} \alpha_{k} \varepsilon^{(m+1) k}=\frac{c_{m} c_{m-1} \ldots c_{1}}{\lambda_{0}^{m}} \quad(m=1,2, \ldots, N) .
$$


If we remark that by definition

$$
\alpha=\alpha(\varepsilon)=\sum_{k=1}^{N-1} \alpha_{k} \varepsilon^{k}=\sum_{k=0}^{N-1} \alpha_{k} \varepsilon^{(N+1) k}
$$

and $\lambda_{0}^{N}=\lambda, c_{N} c_{N_{-1}} \ldots c_{1}=\lambda$, we can write this system as follows:

$$
\sum_{k=0}^{N-1} \alpha_{k} \varepsilon^{(m+1) k}=\frac{c_{m} c_{m-1} \ldots c_{1}}{\lambda_{0}^{m}} \quad(m=1,2, \ldots, N) .
$$

We have obtained the system of $N$ linear non-homogeneous equations with $N$ unknows $\alpha_{1}, \ldots, a_{N}$. The determinant $V$ of the system $(2.9)$ is the Van der Monde determinant of numbers $\varepsilon^{2}, \varepsilon^{3}, \ldots, \varepsilon^{N+1}=\varepsilon$ different one from the others. Hence

$$
V=\prod_{1 \leqslant k, m \leqslant N, k \neq m}\left(\varepsilon^{k}-\varepsilon^{m}\right) \neq 0 .
$$

Let us denote by $V_{k, m}$ the subdeterminant of $V$ obtained by cancelling the $(k+1)$-th column and the $m$-th row. The unique solution of $(2.9)$ is then of the form

$$
a_{k}=\frac{\alpha}{V} \sum_{m=1}^{N} \frac{a_{1} \ldots c_{m}}{\lambda_{0}^{m}} V_{k, m} \quad(k=0,1, \ldots, N-1) .
$$

Since $\alpha$ is an arbitrary complex number, write $d=\alpha / V$ and we obtain the thesis of the theorem if we put

$$
d_{k}=\sum_{m=1}^{N} \lambda_{0}^{-m} c_{1} \ldots c_{m} V_{k, m} .
$$

Now we prove the general theorem without any assumption concerning $\operatorname{dim} \boldsymbol{Z}_{D-\lambda_{0} I}$

THEOREM 2.3 .

$Z_{A}=\left\{z \in X: z=d \sum_{k=0}^{N-1} d_{k} S^{k} z_{0} ; z_{0} \in Z_{D-\lambda_{0} I}\right.$ and the saalar $d$ is arbitrary,

$$
\left.d_{k}=\sum_{m=1}^{N} \lambda_{0}^{-m} c_{1} \ldots c_{m} \nabla_{k, m}\right\}
$$

where we denote by $\nabla_{k, m}$ the subdeterminant obtained by cancelling the $(k+1)$-th column and the $m$-th row of the Van der Monde determinant $V$. of numbers $\varepsilon^{2}, \ldots, \varepsilon^{N}, \varepsilon$ and $c_{m}=a\left(\varepsilon^{m}\right) / b\left(\varepsilon^{m}\right)$.

Proof. Since $Z_{A} \subset Z_{D^{N}-\lambda I}$ (Lemma 2.2), we infer that $z \in Z_{A}$ is of the form

$$
z=\sum_{k=0}^{N-1} S^{k} z_{k}, \quad z_{k} \in Z_{D-\lambda_{0} I}
$$

(Theorem 2.1). If we write $d(S)=-a(S) b^{-1}(S)$, we infer that the equation

$$
A z=[a(S)-b(S) D] z=0
$$

is equivalent to the equation

$$
[D-d(S)] z=0 .
$$

We write

$$
d(S)=\sum_{m=0}^{N-1} d_{m} S^{m}
$$

Since $z \in Z_{D^{N_{-} \lambda I}}$, we have

$$
D z=D \sum_{k=0}^{N-1} S^{k} z_{k}=\sum_{k=0}^{N-1} D S^{k} z_{k}=\sum_{k=0}^{N-1} \varepsilon^{k} S^{k} D z_{k}=\sum_{k=0}^{N-1} \varepsilon^{k} \lambda_{0} S^{k} z_{k} .
$$

Hence, if $z \epsilon Z_{A}$, then

$$
\begin{aligned}
0=[D-d(S)] z & =\sum_{k=0}^{N-1} \varepsilon^{k} \lambda_{0} S^{k} z_{k}-\sum_{k=0}^{N-1} d(S) S^{k} z_{k}=\sum_{k=0}^{N-1} \varepsilon^{k} \lambda_{0} S^{k} z_{k} \\
& =\sum_{k=0}^{N-1} \sum_{m=0}^{N-1} d_{m} S^{m+k} z_{k} .
\end{aligned}
$$

Since $S^{j} z_{k} \in Z_{D-\lambda_{j} I}$ (Theorem 2.1), all elements $S^{j} z_{k}$ are linearly independent. This implies that equation (2.9) is equivalent to the following system equations:

$$
\begin{array}{r}
\left(d_{0}-\lambda_{0}\right) z_{0}+d_{N-1} z_{1}+d_{N-2} z_{2}+\ldots+d_{1} z_{N-1}=0 \\
d_{1} S z_{0}+\left(d_{0}-\varepsilon \lambda_{0}\right) S z_{1}+d_{N-1} S z_{2}+\ldots+d_{2} S z_{N-1}=0
\end{array}
$$
$\ldots \ldots \ldots \ldots \ldots \ldots$ $d_{N-1} S^{N-1} z_{0}+d_{N-2} S S^{N-1} z_{1}+d_{\dot{N}_{-3}} S^{N-1} z_{2}+\ldots+\left(d_{0}-\varepsilon^{N-1} \lambda_{0}\right) S^{N-1} z_{N-1}=0$.

Acting on both sides of the $k$-th equation with the operator $S^{N-k}$ $(k=0,1, \ldots, N-1)$ and using the identity $S^{N}=I$, we obtain the following system of equations:

$$
\begin{array}{r}
\left(d_{0}-\lambda_{0}\right) z_{0}+d_{N-1} z_{1}+\ldots+d_{1} z_{N-1}=0 \\
d_{1} z_{0}+\left(d_{0}-\varepsilon \lambda_{n}\right) z_{1}+\ldots+d_{2} z_{N-1}=0, \\
\ldots \ldots \ldots \ldots \ldots \ldots \ldots \ldots \ldots \ldots \ldots \ldots \ldots \\
d_{N-1} z_{0}+d_{N-2} z_{1}+\ldots+\left(d_{0}-\varepsilon^{N-1} \lambda_{0}\right) z_{N-1}=0 .
\end{array}
$$

Let us consider the matrix $M$ of the system (2.11). First we remark that this matrix does not depend on the dimension of the space $Z_{D-\lambda_{0}} I$. This implies also that the rank of the matrix $M$ does not depend on the dimension of the space $Z_{D-\lambda_{0} I}$. An immediate consequence of Proposition 
2.2 is that rank $M=N-1$ in the case $\operatorname{dim} Z_{D-\lambda_{0} I}=1$. Hence we must have rank $M=N-1$ also in the general case. Further considerations follow the same way as Proposition 2.2.

3. The notation and assumptions of the preceding section remain unchanged. We now determine the general form of solutions of the nonhomogeneous equation $A x=y$.

Lemma 3.1. Let $d(S)=a(S) b^{-1}(S)$. Then

$$
\prod_{m=0}^{N-1} d\left(\varepsilon^{m} S\right)=\lambda I
$$

Proof. Property 1.7 implies

$$
d\left(\varepsilon^{m} S\right)=\sum_{v=1}^{N} d\left(\varepsilon^{m+v}\right) P_{\nu}=\sum_{v=1}^{N} a\left(\varepsilon^{m+\nu}\right) b^{-1}\left(\varepsilon^{m+\nu}\right) P_{v} \quad(m=1,2, \ldots, N-1) .
$$

Property 1.6 implies

$$
\prod_{m=0}^{N-1} d\left(\varepsilon^{m} S\right)=\sum_{\nu=1}^{N}\left[\prod_{m=0}^{N-1} d\left(\varepsilon^{m+\nu}\right)\right] P_{\nu}=\sum_{\nu=1}^{N}\left[\prod_{m=0}^{N-1} \frac{a\left(\varepsilon^{m+\nu}\right)}{b\left(\varepsilon^{n+\nu}\right)}\right] P_{\nu}
$$

We consider the coefficients in the last sum. For $y=1$ we have

$$
\prod_{m=0}^{N-1} \frac{a\left(\varepsilon^{m+1}\right)}{b\left(\varepsilon^{m+1}\right)}=\frac{a(\varepsilon) a\left(\varepsilon^{2}\right) \ldots a\left(\varepsilon^{N}\right)}{b(\varepsilon) b\left(\varepsilon^{2}\right) \ldots b\left(\varepsilon^{N}\right)}=c_{1} c_{2} \ldots c_{N}=\lambda .
$$

For arbitrary $1<\nu \leqslant N$ we obtain a product of $N$ numbers $a\left(\varepsilon^{m+n}\right)$ for $N$ different values $m+v$. Using the equality $\varepsilon^{N+k}=\varepsilon^{k}$ for $k=1,2, \ldots$, $N$, we obtain the product of the same numbers $c_{1}, \ldots, c_{N}$ but in different order for each $v$. Hence

$$
\prod_{m=0}^{N-1} \frac{a\left(\varepsilon^{m+\nu}\right)}{b\left(\varepsilon^{m+\nu}\right)}=\lambda \quad \text { for } \nu=1,2, \ldots, N:
$$

Therefore Corollary 1.1 implies

$$
\prod_{m=0}^{N-1} a\left(\varepsilon^{m} S\right)=\lambda I
$$

Lemma 3.2. Let $\tilde{A}=D^{N-1}+T$, where

$$
\begin{aligned}
T= & d\left(\varepsilon^{N-1} S\right) D^{N-2}+d\left(\varepsilon^{N-1} S\right) d\left(\varepsilon^{N-2} S\right) D^{N-3}+\ldots \\
\text { Then } & +d\left(\varepsilon^{N-1} S\right) \ldots d\left(\varepsilon^{2} S\right) D+ \\
& +d\left(\varepsilon^{N-1} S\right) \ldots d(\varepsilon S) .
\end{aligned}
$$$$
\text { Then }
$$

$$
(D-d(S)) \tilde{A}=\tilde{A}(D-d(S))=D^{N}-\lambda I .
$$

Proof. Let $x \in X$ be arbitrarily fixed and let $u=[d(S)-D] x$. Then $D x=d(S) x-u$.

Acting on both sides of this equation with powers of $D$ and applying Corollary 1.3, we obtain successively:

$$
\begin{gathered}
\begin{aligned}
D^{2} x=D[d(S) x-u]=d\left(\varepsilon S^{\prime}\right) D x-D u & =d(\varepsilon S)[d(S) x-u-D u] \\
& =d(\varepsilon S) d(S) x-d\left(\varepsilon S^{S}\right) u-D u
\end{aligned} \\
\begin{aligned}
D^{3} x=D[d(\varepsilon S) d(S)] x-d(\varepsilon S) u-D u & =d\left(\varepsilon^{2} S\right) d(\varepsilon S) D x-d\left(\varepsilon^{2} S\right) D u-D^{2} u \\
= & d\left(\varepsilon^{2} S\right) d(\varepsilon S)[d(S) x-u]-d\left(\varepsilon^{2} S\right) D u-D u^{2} \\
= & d\left(\varepsilon^{2} S\right) d(\varepsilon S) d(S) x-d\left(\varepsilon^{2} S\right) d(\varepsilon S) u-d\left(\varepsilon^{2} S\right) D u-D u^{2}
\end{aligned}
\end{gathered}
$$

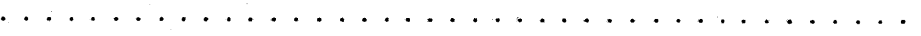

$D^{N} x=d\left(\varepsilon^{N-1} S\right) \ldots d(S) x-\left[d\left(\varepsilon^{N-1} S\right) \ldots d(\varepsilon S)+d\left(\varepsilon^{N-1} S\right) \ldots d\left(\varepsilon^{2} S\right) D+\right.$

$$
\left.+\ldots+d\left(\varepsilon^{N-1} S\right) D^{N-2}+D^{N-1}\right] u \text {. }
$$

Lemma 3.1 implies $D^{N} x=\lambda x-\tilde{A} u$. But $u=[d(S)-D] \dot{x}$. Hence $\left(D^{N}-\lambda I\right) x=-\tilde{A}[\tilde{d}(S)-D] x=\tilde{A}[D-d(S)] x$. Since $x$ was arbitrarily chosen, we find $D^{N}-\lambda I=\tilde{A}[D-d(S)]$.

To prove the first part of formula (3.1), we show that

$$
d(S) \tilde{A}=D T+\lambda I .
$$

Indeed, by Lemma 3.1 and Property 1.8,

$$
\begin{aligned}
d(S) \tilde{A}= & d(S) D^{N-1}+d(S) T \\
= & d\left(\varepsilon^{N} S\right) D^{N-1}+d\left(\varepsilon^{N} S\right) T \\
= & D d\left(\varepsilon^{N-1} S^{\prime}\right) D^{N-2}+d\left(\varepsilon^{N} S\right) D \times \\
& \times\left[d\left(\varepsilon^{N-2} S\right) D^{N-3}+d\left(\varepsilon^{N-2} S\right) d\left(\varepsilon^{N-3}\right) D^{N-4}+\ldots+\right. \\
& \left.+d\left(\varepsilon^{N-2} S\right) \ldots d(\varepsilon S)\right]+d\left(\varepsilon^{N} S\right) d\left(\varepsilon^{N-1} S\right) \ldots d\left(\varepsilon S^{S}\right) \\
= & D\left[d\left(\varepsilon^{N-1} S\right) D^{N-2}+d\left(\varepsilon^{N-1} S\right) d\left(\varepsilon^{N-2} S\right) D^{N-3}+\right. \\
& \left.+\ldots+d\left(\varepsilon^{N-1} S\right) \ldots d(\varepsilon S)\right]+d\left(\varepsilon^{N-1} S\right) \ldots d(\varepsilon S) d(S)=D T+\lambda I
\end{aligned}
$$

On the other hand, by definition

$$
D \tilde{A}=D^{N}+D T
$$

It follows from (3.2) and (3.3) that

$$
[D-d(S)] \tilde{A}=D^{N}+D T-(D T+\lambda I)=D^{N}-\lambda I .
$$

LemaA 3.3. Let $R_{A}=-\tilde{A} b^{-1}(S)$ (where $\tilde{A}$ is determined in Lemma 3.2). Then $R_{d} \tilde{A}=\tilde{A} R_{A}=D^{N}-\lambda I$.

Proof. Since

$D-d(S)=D-a(S) b^{-1}(S)=-b^{-1}(S)[a(S)-b(S) D]=-b^{-1}(S) A$, 
we find

$$
D^{N}-\lambda I=\tilde{A}[D-a(S)]=\tilde{A}\left[-b^{-1}(S) A\right]=\left[-\tilde{A} b^{-1}(S)\right] A=R_{A} A
$$

On the other hand,

$$
D^{N}-\lambda I=[D-d(S)] \tilde{A}=-b^{-1}(S) A \tilde{A} .
$$

Hence $-A \tilde{A}=b(S)\left(D^{N}-\lambda I\right)$. But Property 1.4 implies that $\left(D^{N}-\lambda I\right)$ is commuting with $S$. Therefore $b(S)$ is commuting with $D^{N}-\lambda I$ and $-A \tilde{A}=\left(D^{N}-\lambda I\right) b(S)$. Hence

$$
D^{N}-\lambda I=-A \tilde{A} b^{-1}(S)=A\left[-\tilde{A} b^{-1}(S)\right]=A R_{A} .
$$

From Lemma 3.3 it immediately follows

Proposition 3.1. The operators $A$ and $R_{A}$ are commuting.

LEMMA 3.4. If $\tilde{x}$ is a solution of the equation $\left(D^{N}-\lambda I\right) \tilde{x}=y$, then $x=R_{A} \tilde{x}$ is a solution of the equation $A x=y$.

Indeed, by Lemma 3.3,

$$
A x=A R_{A} \tilde{x}=\left(D^{N}-\lambda I\right) \tilde{x}=y .
$$

Now we can formulate the main theorem:

THEOREM 3.1. Let $S$ be an involution of order $N$ acting in the linear space $X$ (over complex scalars) and let $D$ be permuting $S$. Let $A=a(S)-$ $-b(S) D$, where

$$
a(S)=\sum_{k=0}^{N-1} a_{k} S^{k}, \quad b(S)=\sum_{k=0}^{N-1} b_{k} S^{k}
$$

are polynomials with constant complex coefficients, such that $a\left(\varepsilon^{p}\right) \neq 0$ $\neq b\left(\varepsilon^{v}\right)$ for $v=1,2, \ldots, N$ and $\varepsilon=e^{2 \pi i / N}$. Then every solution of the equation $A x=y$ is of the form

where:

$$
x=R_{A} \tilde{x}+d \sum_{k=0}^{N-1} d_{k} S^{k} z_{0},
$$

$$
\begin{aligned}
& R_{A}=-\left[D^{N-1}+d\left(\varepsilon^{N-1} S\right) D^{N-2}+d\left(\varepsilon^{N-1} S\right) d\left(\varepsilon^{N-2} S\right) D^{N-3}+\right. \\
& a(S)=b^{-1}(S) a(S) \\
& \tilde{x} \text { is a solution of the equation }\left(D^{N}-\lambda I\right) \tilde{x}=y \text {; } \\
& \lambda=\prod_{m=1}^{N} c_{m} \\
& c_{m}=a\left(\varepsilon^{m}\right) / b\left(\varepsilon^{m}\right) \text {; } \\
& d \text { is an arbitrary complex number; } \\
& d_{k}=\sum_{m=1}^{N} \lambda_{0}^{-m} c_{1} \ldots c_{m} V_{k, m}(k=0,1, \ldots, N-1), \quad \lambda_{0}=\sqrt[N]{|\lambda|} e^{\frac{p}{N} i}, \\
& \varphi=\operatorname{Arg} \lambda \quad(0 \leqslant \varphi<2 \pi)
\end{aligned}
$$

$V_{k, m}$ is the subdeterminant obtained by cancelling the $(k+1)$-th cotumn and the $m$-th row of the Van der Monde determinant $V$ of numbers $\varepsilon^{2}, \varepsilon^{3}$, $\ldots, \varepsilon^{N}, \varepsilon^{1}(m=1,2, \ldots, N ; k=0,1, \ldots, N-1)$

$z_{0}$ is an arbitrary solution of the equation $\left(D-\lambda_{0} I\right) z=0$.

The proof immediately follows from Theorem 2.3, Lemmas 3.2, $3.3,3.4$ and from the linearity of the operator $A$.

4. Let $S$ be an involution of order $N$ acting in a linear space $X$ and let $D$ be permuting $S$. Let us consider the operator $A=a(S)-b(S) D$, where $a(S)$ and $b(S)$ are polynomials with constant complex coefficients. In the two last sections we have assumed that $a\left(\varepsilon^{v}\right) \neq 0 \neq b\left(\varepsilon^{\nu}\right)$ for $v=1,2, \ldots, N$. Now we will drop this assumption. We shall consider some most typical cases.

Similarly as in Lemma 2.1, formula (2.3), the equation

$$
A x=y
$$

can be written as an equivalent system of equations

$$
\begin{aligned}
& a\left(\varepsilon^{m}\right) x_{(m)}-b\left(\varepsilon^{m}\right) D x_{(m+1)}=y_{(m)} \quad \text { for } m=1,2, \ldots, N-1, \\
& a\left(\varepsilon^{N}\right) x_{(N)}-b\left(\varepsilon^{N}\right) D x_{(!)}=y_{(N)},
\end{aligned}
$$

where $x_{(m)}=P_{m} x, y_{(m)}=P_{m} y$. Of course, if $a\left(\varepsilon^{m}\right)=b\left(\varepsilon^{m}\right)=0$ for $m=1,2, \ldots, N$, then $A=0$ (Corollary 1.1).

$1^{\circ}$ If $b\left(\varepsilon^{m}\right)=0$ for $m=1,2, \ldots, N$, then the solution of (4.1) was given in [1] (see also [3], p. 89), and it is of the form

$$
x=\sum_{m: a\left(\varepsilon^{m}\right) \neq 0} \frac{1}{a\left(\varepsilon^{m}\right)} P_{m} y+\sum_{m: a\left(\varepsilon^{m}\right)=0} z_{(m)}
$$

(the first sum runs over all $m$ such that $1 \leqslant m \leqslant N$ and $a\left(\varepsilon^{m}\right) \neq 0$, the second one over all $m$ such that $1 \leqslant m \leqslant N$ and $\left.a\left(\varepsilon^{m}\right)=0\right)$ under the necessary and sufficient condition

$$
P_{m} y=0 \quad \text { for all } m \text { such that } a\left(\varepsilon^{m}\right)=0,
$$

where $z_{(m)}$ is an arbitrary element of the space $X_{(m)}=P_{m} X$.

$2^{\circ}$ If $a\left(\varepsilon^{m}\right)=0$ for $m=1,2, \ldots, N$, then we solve equation (4.1) with respect to the unknown $D x$. We reduce our problem (similarly as in $\left.1^{\circ}\right)$ to the equation

$$
D x=y_{0}
$$

where

$$
y_{0}=-\sum_{m: b\left(\varepsilon^{m}\right) \neq 0} \frac{1}{b\left(\varepsilon^{m}\right)} P_{m} y-\sum_{m: b\left(\varepsilon^{m}\right)=0} z_{(m)} \quad(m=1,2, \ldots, N),
$$

Studia Mathematica XXXV.1 
under the necessary and sufficient condition $P_{m} y=0$ for all $m$ such that $b\left(\varepsilon^{m}\right)=0$, and $z_{(m)} \epsilon X_{(m)}$ are arbitrary.

$3^{\circ}$ Let us suppose that $a\left(\varepsilon^{m}\right) \neq 0$ for all $m$ and $b\left(\varepsilon^{m}\right)=0$ for at least one $m$. Without loss of generality we can consider the case $b\left(\varepsilon^{N}\right)=0$. From the last equation of $(4.2)$ we obtain $x_{(N)}=a^{-1}\left(\varepsilon^{N}\right) y_{(N)}$, and solving the system (4.2) successively, we have

$$
\begin{aligned}
& x_{(N)}=\frac{1}{a\left(\varepsilon^{N}\right)} y_{(N)}, \\
& x_{(m)}=\frac{1}{a\left(\varepsilon^{m}\right)} y_{(m)}+\frac{b\left(\varepsilon^{m}\right)}{a\left(\varepsilon^{m}\right)} D x_{(m+1)} \quad(m=1,2, \ldots, N-1) .
\end{aligned}
$$

Hence, by Properties 1.2 and 1.3,

$$
\begin{aligned}
x_{(m)} & =\frac{1}{a\left(\varepsilon^{m}\right)}\left[y_{(m)}+\frac{b\left(\varepsilon^{m}\right)}{a\left(\varepsilon^{m+1}\right)} D y_{(m+1)}+\ldots+\frac{b\left(\varepsilon^{m}\right) \ldots b\left(\varepsilon^{N-1}\right)}{a\left(\varepsilon^{m+1}\right) \ldots a\left(\varepsilon^{N}\right)} D^{N-m} y_{(N)}\right] \\
& =\frac{1}{a\left(\varepsilon^{m}\right)}\left[P_{m}+\frac{b\left(\varepsilon^{m}\right)}{a\left(\varepsilon^{m+1}\right)} D P_{m+1}+\ldots+\frac{b\left(\varepsilon^{m}\right) \ldots b\left(\varepsilon^{N-1}\right)}{a\left(\varepsilon^{m+1}\right) \ldots a\left(\varepsilon^{N}\right)} D^{N-m} P_{N}\right] y \\
& =\frac{1}{a\left(\varepsilon^{m}\right)} P_{m}\left[I+\frac{b\left(\varepsilon^{m}\right)}{a\left(\varepsilon^{m+1}\right)} D+\ldots+\frac{b\left(\varepsilon^{m}\right) \ldots b\left(\varepsilon^{N-1}\right)}{a\left(\varepsilon^{m+1}\right) \ldots a\left(\varepsilon^{N}\right)} D^{N-m}\right] y
\end{aligned}
$$

and

$$
\begin{aligned}
x & =\sum_{m=1}^{N} x_{(m)} \\
& =\sum_{m=1}^{N} \frac{1}{a\left(\varepsilon^{m}\right)} P_{m}\left[I+\frac{b\left(\varepsilon^{m}\right)}{a\left(\varepsilon^{m+1}\right)} D+\ldots+\frac{b\left(\varepsilon^{m}\right) \ldots b\left(\varepsilon^{N-1}\right)}{a\left(\varepsilon^{m+1}\right) \ldots a\left(\varepsilon^{N}\right)} D^{N-m}\right] y .
\end{aligned}
$$

In a similar way we determine the solution of $(4.2)$ if $b\left(\varepsilon^{m}\right)=0$ for an $m \neq N$.

$5^{0}$ Let us suppose that $b\left(\varepsilon^{m}\right) \neq 0$ for all $m$ and $a\left(\varepsilon^{m}\right)=0$ for at least one $m$. As previously, we consider the case $a\left(\varepsilon^{N}\right)=0$. Then we determine $x_{(1)}$ from the equation

$$
D x_{(1)}=-\frac{1}{b\left(\varepsilon^{N}\right)} y_{(N)}
$$

obtained from the last equation (4.2). Having $x_{(1)}$, we successively solve the equations

$$
D x_{(m+1)}=\frac{-1}{b\left(\varepsilon^{m}\right)} y_{(m)}+\frac{a\left(\varepsilon^{m}\right)}{b\left(\varepsilon^{m}\right)} x_{(m)} \quad(m=1,2, \ldots, N-1)
$$

obtained from the first $N-1$ equations (4.2). Similarly, we solve equation (4.1) if $a\left(\varepsilon^{m}\right)=0$ for an $m \neq N$

5. Example. Let us consider on the complex plane the differential equation

$$
\sum_{k=0}^{N-1} a_{k} x\left(\varepsilon^{k} t+\beta_{k}\right)+\sum_{k=0}^{N-1} b_{k} x^{\prime}\left(\varepsilon^{k} t+\beta_{k}\right)=y(t),
$$

where $a_{k}, b_{k}, \beta_{k}$ are constant complex numbers and $\varepsilon=e^{2 \pi i / N}, N \geqslant 2$. Let us consider the following operator:

$$
(S x)(t)=x\left(\varepsilon t+\beta_{0}\right) .
$$

It is an involution of order $N$ in the space of all functions of one complex variable. Indeed, it is easy to check that

$$
\left(S^{m} x\right)(t)=x\left(\varepsilon^{m} t+\beta_{0}\left(\varepsilon^{m-1}+\varepsilon^{m-2}+\ldots+\varepsilon+1\right)\right) .
$$

Hence

$$
\left(\mathcal{S}^{N} x\right)(t)=x\left(\varepsilon^{N} t+\beta_{0}\left(\varepsilon^{N-1}+\ldots+\varepsilon+1\right)\right) .
$$

But $\varepsilon^{N}=1$ and, by formula $(1.3), \varepsilon^{N-1}+\ldots+\varepsilon+1=0$. Then $\left(S^{N} x\right)(t)=x(t)$.

The differentiation operator is permuting $S$. Indeed,

$$
(D S x)(t)=\left[x\left(\varepsilon t+\beta_{0}\right)\right]^{\prime}=\varepsilon x^{\prime}\left(\varepsilon t+\beta_{0}\right)=\varepsilon(S D x)(t) .
$$

Hence all previous considerations can be applied to equation (5.1) if we assume additionally, according to (5.2), that

$$
\beta_{k}=\dot{\beta}_{0}\left(\varepsilon^{k-1}+\ldots+\varepsilon+1\right) \quad \text { for } k=1,2, \ldots, N-1 .
$$

For example, if

$$
a\left(\varepsilon^{m}\right)=\sum_{k=0}^{N-1} a_{k} \varepsilon^{m k} \neq 0 \neq b\left(\varepsilon^{m}\right)=\sum_{k=0}^{N-1} b_{k} \varepsilon^{m 2 k} \quad(m=1,2, \ldots, N),
$$

according to Theorem 3.1, to solve equation (5.1) it is sufficient to know all solutions of the equation $z^{\prime}-\lambda_{0} z=0$ and a solution of the equation $\tilde{x}^{(N)}-\lambda \tilde{x} \doteq y$, where

$$
\lambda=\prod_{m=1}^{N} a\left(\varepsilon^{m}\right) / b\left(\varepsilon^{m}\right)
$$

and

$$
\lambda_{0}=\sqrt[n]{|\lambda|} e^{\varphi i / N}, \quad \varphi=\operatorname{Arg} \lambda .
$$




\section{References}

[1] D. Przeworska-Rolewicz, Sur les involutions d'ordre n, Bull. Acad. Pol. Sc. 8 (1960), p. $735-739$.

[2] - On equations with reflection, Studia Math. 33 (1969), p. 191-199.

[3] - and S. Rolewicz, Equations in linear spaces, Warszawa 1968.

INSTITUTE OF MATHEMATICS OF THE POLISH ACADEMY OF SCIENCES INSTYTUT MATEMATYCZNY POLSKIEJ AKADIMII NAUK

Reçu par la Rédaction le 4. 3. 1969

\section{On an equation with reflection of order $n$}

by

\section{BARBARA MAŻBIC-KULMA (Warszawa)}

If a differential equation contains together with the unknown function $x(t)$ the function $x(-t)$, then it is called a differential equation with reflection.

D. Przeworska-Rolewicz gives in [1] the general solution of an equation with reflection of order 1 , i.e. of the equation

$$
a_{0} x(t)+b_{0} x(-t)+a_{1} x^{\prime}(t)+b_{1} x^{\prime}(-t)=y(t),
$$

where $a_{0}, a_{1}, b_{0}$ and $b_{1}$ are scalars.

In the present paper we consider the differential equation with reflection of order $n$,

$$
a_{0} x(t)+b_{0} x(-t)+\ldots+a_{n} x^{(n)}(t)+b_{n} x^{(n)}(-t)=y(t),
$$

where the coefficients $a_{0}, \ldots, a_{n}, b_{0}, \ldots, b_{n}$ are constants. We give a general form of the solution of (1) under the following assumptions:

$$
1^{0} a_{n}^{2}-b_{n}^{2} \neq 0 \text {; }
$$

$2^{0} a_{j-k} a_{k}-b_{j-k} b_{k} \neq 0(k=0,1, \ldots, n$ and $j=k+1, \ldots, k+n)$;

$3^{\circ}$ the polynomial $\sum_{j=0}^{n} \lambda_{2 j} t^{j}$ has single roots only for $k=0,1, \ldots, n$, where

$$
\lambda_{j}= \begin{cases}\sum_{k=0}^{j} c_{j k} & \text { for } 0 \leqslant j \leqslant n \\ \sum_{k=j-n}^{n} c_{j k} & \text { for } n<j \leqslant 2 n\end{cases}
$$

$$
c_{j k}=(-1)^{j-k}\left(a_{j_{-k}} a_{k}-b_{j_{-k}} b_{k}\right)\left(a_{n}^{2}-b_{n}^{2}\right)^{-1} .
$$

1. Let $S$ be a reflection: $S x(t)=x(-t)$. Since $S^{2}=I$, where $I$ is the identity operator, $S$ is an involution. We write$$
D x(t)=x^{\prime}(t) .
$$ 